\title{
Fentanyl not detected in routine urine drug screens: case report
}

\author{
Dominick Shelton ${ }^{1}$ (1)
}

Received: 15 July 2021 / Accepted: 20 January 2022 / Published online: 9 February 2022

(C) The Author(s), under exclusive licence to Canadian Association of Emergency Physicians (CAEP)/ Association Canadienne de Médecine d'Urgence (ACMU) 2022

Keywords Fentanyl $\cdot$ Urine drug screen · Emergency department

\section{Introduction}

The opioid epidemic has led to frequent illicit use of fentanyl that is knowingly and unknowingly consumed. Despite this, many hospitals' urine drug screen for drugs of abuse do not detect fentanyl. Like oxycodone and methadone, fentanyl is not metabolized to morphine, and so requires a separate assay. Many emergency physicians may be surprised to learn that urine toxicology screens conducted in their hospital may not detect fentanyl. While in the midst of a North American opioid epidemic where fentanyl is the illicit drug of choice, it is important that physicians are not falsely reassured by a negative urine drug screen for opioids. The goal of sharing this case report is for emergency physicians to be aware of this limitation of urine drug screens and determine if it applies to their hospital.

\section{Clinical scenario}

A 64-year-old male was brought to the emergency department (ED) as a code stroke due to decrease level of consciousness, slurred speech and possible right arm weakness. He was last seen normal approximately $2 \mathrm{~h}$ prior to ED presentation. The patient was recently diagnosed with pancreatic cancer and was awaiting surgical treatment. He was taking acetaminophen for mild abdominal pain.

His vital signs were: temperature 37.4, pulse 102, RR 12, BP 105/64, Sa02 92\%

His Glasgow coma scale was 8 (eyes 2, verbal 2, motor 4). Pupils were $3 \mathrm{~mm}$, equal and reactive. He was not

Dominick Shelton

Dominick.shelton@sunnybrook.ca

1 Sunnybrook Health Sciences Centre, University of Toronto, 2075 Bayview Avenue, Toronto, ON M4N 3M5, Canada responding to verbal commands and withdrew both arms to painful stimulus. Chest exam was clear. Cardiovascular exam revealed normal heart sounds and no murmurs. On abdominal exam, he was mildly distended with no palpable masses and no painful response on palpation.

Point of care glucose was 7.1 and ECG revealed normal sinus rhythm at $96 \mathrm{bpm}$. The decision was made to intubate the patient prior to obtaining a CTA head and neck. While preparing for intubation, naloxone $0.4 \mathrm{mg} I V$ was given due to decrease level of consciousness. Unexpectedly, the patient awoke after receiving naloxone. He verbalized normally, answered questions appropriately and moved all limbs with normal power. The patient denied taking any opioids or illicit drugs, but admitted to smoking marijuana on the same day that he purchased illegally.

His routine blood investigations were all normal. Urine drug screen was positive for cannabinoids, but negative for all other drugs (including opiates, oxycodone and methadone). The patient was admitted to hospital for observation and to further evaluate the cause of his presentation. His blood and urine samples were sent to the regional toxicology centre and his broad spectrum urine drug screen was positive for fentanyl.

\section{Discussion}

Although screening urine for drugs of abuse in the ED rarely affects patient management [1], there are select patients where it provides valuable information, like the case described. When there is a discrepancy between the clinical presentation of a toxidrome and no apparent source, urine drug screen may assist in confirming exposure. However, it is important to realize the limitations of urine drug screens, including the inability to detect fentanyl.

Most hospitals' routine urine toxicology screens for drugs of abuse use a standard opiate screen that detects morphine, 
a metabolite of heroin and codeine, but not fentanyl or its analogs [2]. Fentanyl, oxycodone, and methadone are not metabolized to morphine, so require separate detection in urine testing [3].

Fentanyl is a synthetic opioid that is about 50 times as potent as heroin and is more prevalent because it is cheap and requires only a small quantity [4]. As the opioid epidemic has evolved, fentanyl has replaced heroin as the illicit drug of choice [2]. However, routine urine toxicology screening has not adapted to this change to detect the current most prevalent opioid of abuse.

In 2018 , a study was conducted to determine the prevalence of illicit fentanyl use in ED patients seeking care for drug overdose. They found that of patients who had both standard hospital urine drug screen and point of care fentanyl urine strip testing, 56\% were positive for fentanyl on urine strips and negative for opiates on urine drug screen. This study highlighted that fentanyl was common and frequently missed amongst ED patients. They recommended that hospitals should add fentanyl to their urine drugs of abuse panel [2].

In light of the above case, a request was made to the head of hospital chemistry laboratory at the author's hospital that fentanyl testing be routinely included in urine drug screen. The request was favorably met by sourcing a new urine drug screen cartridge panel that detects fentanyl for an additional cost of \$2.50 per test. Since this updated urine drug screen has a quick turn-around time, it can also be used in addiction medicine clinics.

An alternative approach in testing for fentanyl is to order an extended urine drug screen at a regional toxicology centre. This approach is lengthy and often impractical to impact ED patient management.

\section{Conclusion}

Urine drug screen has not been updated in many hospitals, as the opioid epidemic has evolved, to detect the most common illicit opioid, fentanyl. This clinical scenario is presented to demonstrate this important limitation of in-hospital urine drug screen and approaches to test for fentanyl when indicated.

\section{Declaration}

Conflict of interest The author have no conflicts of interest to declare.

\section{References}

1. Eisen JS, et al. Screening urine for drugs of abuse in the emergency department: do test results affect physicians' patient care decisions? CJEM. 2004;6(2):104-11.

2. Dezman ZDW, Feleban W, Bontempo L, et al. Evidence of fentanyl use is common and frequently missed in a cross-sectional study of emergency department patients in Baltimore. Maryland Clin Toxicol. 2020;58(1):59-61.

3. Moeller KE, Lee KC, Kissack JC. Urine drug screening: practical guide for clinicians. Mayo Clin Proc. 2008;83(1):66-76.

4. Harm Reduction Coalition (2022). https://harmreduction.org/ issues/fentanyl/. Accessed 2 June 2021 